\title{
Influence of Infinitesimal Neglected Effects by Current Theory of Gravitation and Experiments on the Stability of the Universe
}

\author{
Ping'an Luo \\ Room 21-2-502, Block 3, Huilong Guan Yunqu Estate, Beijing, China \\ Email: luopingan6362@163.com, luopingan6362@sohu.com
}

Received 30 May 2014; revised 28 June 2014; accepted 21 July 2014

Copyright (C) 2014 by author and Scientific Research Publishing Inc. This work is licensed under the Creative Commons Attribution International License (CC BY). http://creativecommons.org/licenses/by/4.0/

(c) (i) Open Access

\section{Abstract}

From the hypotheses compatible with microphysics theory, this paper establishes a new theoretical model of static universal gravitation and deduces new formula of the theory of universal gravitation. In a first order approximation, the new formula shows the inverse-square law consistent with Newton formula, which would indicate that the new theory is consistent with the experimental results that can be reasonably explained by the current theory of gravitation. The parameters and higher order terms among the coefficients of this paper reveal the numerous infinitesimal neglected effects by current theory and experiments. In the first order approximation, the meanings of the physical parameters included in coefficients are analyzed and the infinitesimal neglected effects are applied in the study of the stability of the universe, which overcomes the difficulty of singularity in the cosmology of Newton, Einstein, etc., and concludes that the boundary of universe is unlimited, without any need of the hypothesis that the universe starts off with the big bang. Therefore, this paper establishes a harmonious and ingenious relationship between microphysics and macrophysics theories. In addition, through the analysis of the formula derived from the theory of this paper, it is found that: in general, the gravitational constant is not always a constant in the gravitation formula requiring high precision; from the perspective of the interaction of field quantum, the acting force may not be equal to counter-acting force under the interaction of indirect contact; the gravity process is an exothermic process; in the gravitational process, annihilation effects may exist amongst gravitons; reciprocal translation may exist amongst fundamental forces.

\section{Keywords}

Universal Gravitation, Graviton, Annihilation Effect, General Theory of Relativity, Steady-State Model of the Universe, Infinitesimal 


\section{Introduction}

Since Newton proposed the law of universal gravitation in the Philosophiae Naturalis Principia Mathematica, people have extended their knowledge by applying the theory of gravitation, and have also tried many times to revise the law. For example, Newcomo, et al. [1] proposed the inverse-square law in Newtons law of universal gravitation, Poincare [2] revised the instantaneous action at a distance by postponing $t=r / c$, and Einstein proposed the general theory of relativity-gravitation theory. With regards to Chinese national research, till now, lots of researchers have been engaged in the revision, and Lv Jiahong directly revised Newton's law of universal gravitation with relativity theory [3] [4]. As for foreign research, there were also researchers trying to find the fifth kind of basic acting force [5] by measuring the change of the gravitation constant, (USA) R. D. Newman proposed a coefficient formula $G(r)=G_{\infty}\left(1+\alpha \mathrm{e}^{-r / \lambda}\right)$ [6] through experiments. Although lots of revisions have been conducted, the following problems still haven't been solved:

1) In 1686, influenced by the then classical concept of action at a distance, Newton thought that the universal gravitation of $M$ on $m$ equals to that of $m$ to $M$ (maybe influenced by his Third Law of Motion). However, in the age of Quantum Field Theory, do Newton's thoughts still stand? The answer may surprise us. However, up until now there are no reports on the revision of this aspect.

2) Newton's theory leads to a difficulty in cosmology. According to Newton's theory, the number of "lines of force” from infinity to mass $m$ is directly proportional to mass $\mathrm{m}$. If on average the mass density $\rho$ is a constant in the whole universe, the sphere with the volume of $V$ has average mass $\rho V$. Therefore, the number of lines of force passing through a spherical surface $S$ and entering into the sphere is directly proportional to $\rho V$. As for unit sphere area, the lines of force into a sphere are directly proportional to $\rho V / S$ or $\rho R$. Therefore, as the semi-diameter of sphere $R$ increases, the field intensity of the sphere will become infinite, which is impossible [7]. Although Hugo von Seeliger revised the problem, the revision and its complexity have no experimental and theoretical basis.

3) The problem of Einstein's general theory of relativity on cosmology. According to the general theory of relativity, the relationship between spatial scale and mean substance density of the universe can be simply expressed as:

$$
R^{2}=\frac{2}{k \rho}
$$

where, in $\mathrm{cm} \cdot \mathrm{g} \cdot \mathrm{s}$ system, $2 / \mathrm{k}=1.08 \times 10^{27}$ is obtained; $\rho$ is mean density, $k$ is a constant relating to the gravitational constant [8].

The actual mean universe density will never be zero no matter how small it is. Therefore, $R$ is limited, no matter if the universe is torispherical or quasi-ellipsodial, which indicates the universe is in a limited space. Einstein said that it was impossible to establish boundary conditions which opposes "the theory of extinction" and confirms sphere rate is low, just as W. Pauli said “cosmic space is bounded” [9].

However, there remains a problems, what is it outside the "bounded space”?

4) The method of geometrization of the gravitational field adopted in the General theory of relativity causes a man-made gap between gravitation and other interactions [10] and makes the problem abstract and complicated.

5) Previous literatures [11] pointed out: "Physicists also realized that, before a complete explanation about the motion of matter is given, a core problem shall be solved. The problem is the incompatibility between macrophysics and microphysics."

6) Document [12] summarizes the current popularized Big Bang theory involved in the dark matter and energy, which proposed that:

By fitting a theoretical model of the composition of the Universe to the combined set of cosmological observations, scientists have come up with the composition that we described above, $\sim 68 \%$ dark energy, $27 \%$ dark matter, 5\% normal matter. And the document [13] also proposes that the arguments on the issue of dark energy are still existent, even though there are different opinions about it. The key point that caused this situation lies in other existing explanations on the Cosmic Explosion. For instance, in the largest scale of the universe, there is a big difference between the action mode and expression of the gravitation and the general situation.

Even though the scientists have devoted large amounts of manpower and material resources, the prophetic dark matter and energy by the explosion theory from the experiments are still not found so far. If it exists a new gravitation theory implying the evolution of the universe, which does not depend on the dark matter and energy, and will the theory of physics be more simple, understandable and researchable? 
7) To simplify work, scientists always neglect the influence of less important causes in experiments and studies of theoretical problems, which is effective in practical work. However, it is a problem whether those less important causes are neglected in study of infinite space. From the perspective of mathematics, the sum of limited infinitesimal is infinitely small, but the sum of unlimited infinitesimal is not always infinitely small, which may be infinitely great or a constant. Currently, the known gravitation theory and experiments are based on experiences about limited space. It will be a problem whether the less important causes are still neglected once the theory and experiments are expanded to infinite space, and whether the constants can still work as constants in infinite space is also a problem.

For example: to simplify the workload, most physical models extract the object as mass point [14] in modeling and experimental studies. Such approximation shows as approximate or exact in the studies in which objects are few and make no difference by volume, because what is neglected is limited infinitesimal. Once the conclusion extends to studies with many or unlimited objects, problems will be caused, because the sum of unlimited infinitesimal is not always infinitely small.

Previous literatures [15] pointed out that, the famous modern astrophysicist F. Hoyle and other researchers strongly advocated steady-state cosmological model and made lots of studies. But, it is difficult to establish a convincing steady-state cosmological model if the defects in Newton's theory of universal gravitation and Einstein's general theory of relativity are not found.

All in all, the current many gravitational theories are all generalized conclusions from the experiences or facts which are based on small scale space. If extending these theories in infinite space without preconditions, it is unavoidable to come across difficulties. Therefore, it is undoubtedly significant to start from a new perspective, apply the basic concepts compatible with microphysics, strictly deduce static-steady gravitation formula (macrophysics) in theory, express the neglected or unclear causes in previous theories and experiments, and make clear the physical significance and the change laws of each parameter.

\section{Theoretical Derivation}

For simplifying the derivation process of the theory of universal gravitation, only the static interaction between two spherical objects is taken into consideration.

Basic hypotheses:

1) Field quantum of universal gravitation (graviton) has the same emission probability in all aspects, or an emission parent including a large quantity of gravitons has the same emission probability in multiple directions.

2) The overall acting force of a graviton on an object is approximately proportional to the total flux absorbed by the object.

3) The contribution of each graviton to the acting force of matter is approximately the same.

4) As for the matter in an equilibrium position, the changes in internal structure caused by the acting force of gravitons can be neglected.

5) The probability of an object giving off gravitons will not be influenced by the gravitons received, and is only related to its own characteristics.

Based on the above hypotheses, the action of universal gravitation of the two spherical objects is inferred.

The features of study object are as follows (Table 1).

The distance between the centers of mass (the distance between the two sphere centers) is $r$. See Figure 1.

Assume the flux of the graviton given off by the object with mass differential of $\mathrm{dm}$ is:

$$
\mathrm{d} \phi=a \cdot \mathrm{d} m
$$

where, $a$ is a constant for $\mathrm{dm}$.

Firstly, infer the number $n_{m \rightarrow M}$ of gravitons received by $M$ from $m$ in the unit time. Establish a coordinate system according to Figure 2.

Randomly take a differential dm from $m$, set its coordinate as $\left(r_{2}, \varphi, \theta\right)$, coordinate of $M$ center $(r, 0,0)$.

$$
\because \text { the solid angle of } M \text { for } m \text { is } \frac{s}{r_{1}^{2}-R_{M}^{2}}
$$

Note: $s$ is the formed areas by the intersect of $M$ and the spherical surface that is taking dm for the center of the sphere and $\sqrt{r_{1}^{2}-R_{M}^{2}}$ spherical radius. See Figure 4 . 


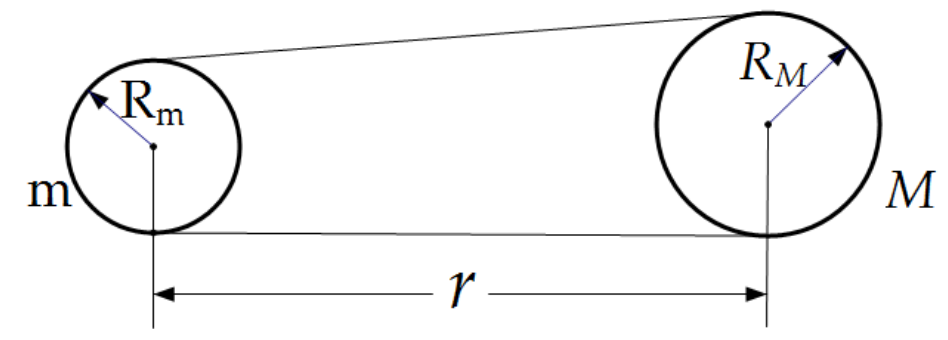

Figure 1. Positions of $m$ and $M$ Spheres.

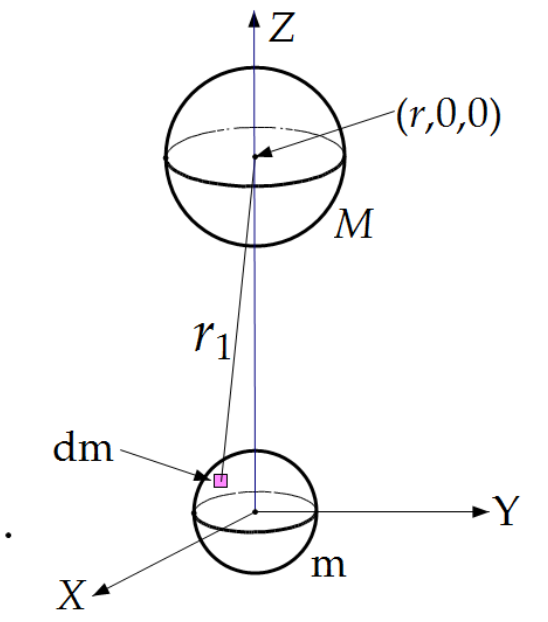

Figure 2. Establishment of coordinate system.

Table 1. Letters for object features.

\begin{tabular}{ccc}
\hline & The first object & The second object \\
\hline Mass & $M$ & $m$ \\
Density & $\rho_{M}$ & $\rho_{m}$ \\
Volume & $V_{M}$ & $V_{m}$ \\
Semi-diameter & $R_{M}$ & $R_{m}$ \\
\hline
\end{tabular}

See Figure 3:

$$
\begin{gathered}
\sin \alpha=\frac{R_{M}}{r_{1}} \\
\cos \alpha=\frac{\sqrt{r_{1}^{2}-R_{M}^{2}}}{r_{1}}
\end{gathered}
$$

The spherical crown intersected by $M$ and the sphere with the semi-diameter of $\sqrt{r_{1}^{2}-R_{M}^{2}}$ See Figure 4.

$$
h=\sqrt{r_{1}^{2}-R_{M}^{2}}(1-\cos \alpha)=\sqrt{r_{1}^{2}-R_{M}^{2}}\left(1-\frac{\sqrt{r_{1}^{2}-R_{M}^{2}}}{r_{1}}\right)
$$




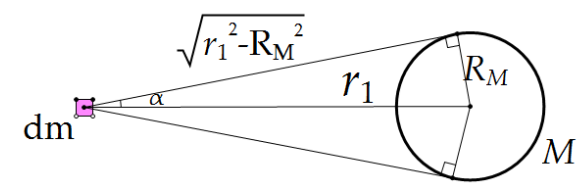

Figure 3. Relationships among parameters in differential model.

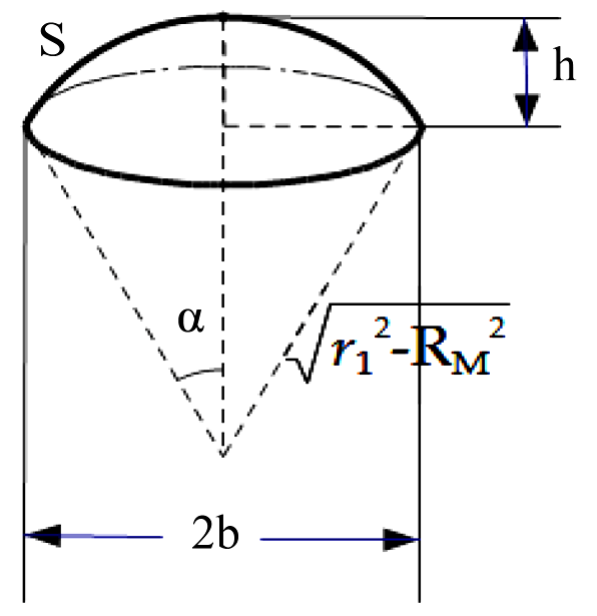

Figure 4. Relationships among parameters of spherical crown.

$$
\begin{aligned}
\therefore s= & 2 \pi \sqrt{r_{1}^{2}-R_{M}^{2}} h=2 \pi \sqrt{r_{1}^{2}-R_{M}^{2}}\left[\sqrt{r_{1}^{2}-R_{M}^{2}}\left(1-\frac{\sqrt{r_{1}^{2}-R_{M}^{2}}}{r_{1}}\right)\right] \\
= & 2 \pi\left(r_{1}^{2}-R_{M}^{2}\right)\left(1-\sqrt{1-\left(\frac{R_{M}}{r_{1}}\right)^{2}}\right) \\
\therefore \mathrm{d} n_{m \rightarrow M} & =\frac{a \cdot \mathrm{d} m}{4 \pi} \cdot \frac{s}{r_{1}^{2}-R_{M}^{2}}=\frac{a \cdot \rho_{m} \cdot \mathrm{d} v_{m}}{4 \pi} \cdot \frac{2 \pi\left(r_{1}^{2}-R_{M}^{2}\right)\left(1-\sqrt{1-\left(\frac{R_{M}}{r_{1}}\right)^{2}}\right)}{r_{1}^{2}-R_{M}^{2}} \\
& =\frac{a \cdot \rho_{m} \cdot \mathrm{d} v_{m}}{2}\left(1-\sqrt{1-\left(\frac{R_{M}}{r_{1}}\right)^{2}}\right)
\end{aligned}
$$

Based on the above coordinate system, the following formula (see Figure 5) can be obtained:

$$
r_{1}^{2}=r^{2}+r_{2}^{2}-2 r r_{2} \cos \theta
$$

Put it into the formula above, it is obtained about $V_{m}$ integration

$$
\begin{aligned}
n_{m \rightarrow M} & =\iiint_{V_{m}} \frac{a \cdot \rho_{m}}{2}\left(1-\sqrt{1-\left(\frac{R_{M}}{r_{1}}\right)^{2}}\right) \mathrm{d} v_{m} \\
& =\iiint_{V_{m}} \frac{a \cdot \rho_{m}}{2}\left(1-\sqrt{1-\frac{R_{M}^{2}}{r^{2}+r_{2}^{2}-2 r r_{2} \cos \theta}}\right) \mathrm{d} v_{m}
\end{aligned}
$$




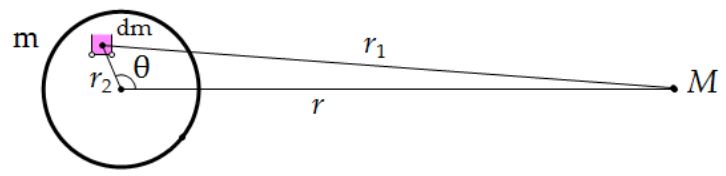

Figure 5. Relationships among parameters between $M$ and differential model.

Considering the self-absorption of graviton by $m$ and the probability of the actions between graviton and $M$, the total self-absorption coefficient $\alpha_{1 m}$ of $m$ and the total self-absorption coefficient $\alpha_{2 M}$ of $M$ is put in.

The definition of the total self-absorption coefficient $\alpha_{1 m}$ of $m$ is:

$$
\alpha_{1 m}=\frac{\text { Total quantity of gravitons given off from } m \text {-the quantity of gravitons that go off } m \text { surface }}{\text { Total quantity of gravitons given off from } m}
$$

Because any differential in $m$ has attractive interactions to others, in other words, some of the gravitons which are emitted by any one of the differentials in the $m$ are absorbed by other differential, and the total absorption coefficient is a parameter to quantitatively describe this total effect.

The definition of the total absorption coefficient $\alpha_{2 \mathrm{M}}$ of $M$ is:

$$
\alpha_{2 M}=\frac{\text { The quantity of gravitons that entered } M \text { and be received by } M}{\text { The quantity of gravitons that entered } M}
$$

Similarly, in general, $M$ is impossible to totally absorb the all gravitons which is from $m$, and the total absorption coefficient is a parameter to quantitatively describe this total effect. For example, an object which in a house is not immune gravity even through it is isolated by the house. That is to say that the absorption of gravitons by this object has an absorption probability and its macro performance is the absorption coefficient.

So, $0 \leq \alpha_{1 m}<1,0 \leq \alpha_{2 M} \leq 1$. It needs noting that the subscript 1 represents total self-absorption coefficient, the subscript 2 represents total absorption coefficient; subscript $m$ represents the physical process in the object with mass of $m$, and the subscript $M$ represents the physical process in the object with mass of $M$.

$$
n_{m \rightarrow M}=\frac{a\left(1-\alpha_{1 m}\right) \alpha_{2 M}}{2} \int_{0}^{2 \pi} \mathrm{d} \varphi \int_{0}^{\pi} \sin \theta \mathrm{d} \theta \int_{0}^{R_{m}} \rho_{m}\left(1-\sqrt{1-\left(\frac{R_{M}^{2}}{r^{2}+r_{2}^{2}-2 r r_{2} \cos \theta}\right)}\right) r_{2}^{2} \mathrm{~d} r_{2}
$$

Set $\rho_{m}(r, \varphi, \theta)$ approximately to constants, so,

$$
n_{m \rightarrow M}=\pi a\left(1-\alpha_{1 m}\right) \alpha_{2 M} \rho_{m} \int_{0}^{\pi} \sin \theta \mathrm{d} \theta \int_{0}^{R_{m}}\left(1-\sqrt{1-\frac{R_{M}^{2}}{r^{2}+r_{2}^{2}-2 r r_{2} \cos \theta}}\right) r_{2}^{2} \mathrm{~d} r_{2}
$$

the force from $m$ to $M$ is:

$$
F_{M}=n_{m \rightarrow M} f=\pi a\left(1-\alpha_{1 m}\right) \alpha_{2 M} \rho_{m} f \int_{0}^{\pi} \sin \theta \mathrm{d} \theta \int_{0}^{R_{m}}\left(1-\sqrt{1-\frac{R_{M}^{2}}{r^{2}+r_{2}^{2}-2 r r_{2} \cos \theta}}\right) r_{2}^{2} \mathrm{~d} r_{2}
$$

Here $f$ as the acting force of a graviton that on average contributes to $M$ along the line of mass center. (The sum of component forces in other directions is zero.) Set

$$
g\left(R_{m}\right)=\int_{0}^{R_{m}}\left(1-\sqrt{1-\frac{R_{M}^{2}}{r^{2}+r_{2}^{2}-2 r r_{2} \cos \theta}}\right) r_{2}^{2} \mathrm{~d} r_{2}
$$

$\omega\left(R_{m}\right)=r^{2}+R_{m}^{2}-2 r R_{m} \cos \theta$

Method 1:

Under the condition of $r \gg R_{M}+R_{m}$, extend Formula (2) based on the formula below 


$$
\begin{gathered}
g(x)=g(0)+\frac{\frac{\mathrm{d} g(0)}{\mathrm{d} x}}{1 !} x+\frac{\frac{\mathrm{d}^{2} g(0)}{\mathrm{d} x^{2}}}{2 !} x^{2}+\cdots \\
\because \frac{\mathrm{d} g}{\mathrm{~d} R_{m}}\left(R_{m}\right)=\left(1-\sqrt{1-\frac{R_{M}^{2}}{r^{2}+R_{m}^{2}-2 r R_{m} \cos \theta}}\right) R_{m}^{2}=\left(1-\sqrt{1-\frac{R_{M}^{2}}{\omega}}\right) R_{m}^{2} \\
\text { So }\left.\frac{\mathrm{d} g\left(R_{m}\right)}{\mathrm{d} R_{m}}\right|_{R_{m}=0}=0 \\
\frac{\mathrm{d}^{2} g\left(R_{m}\right)}{\mathrm{d} R_{m}^{2}}=2 R_{m}\left(1-\sqrt{1-\frac{R_{M}^{2}}{\omega}}\right)+R_{m}^{2}\left(-\frac{-\frac{R_{M}^{2}}{\omega^{2}} \omega^{\prime}}{2 \sqrt{1-\frac{R_{M}^{2}}{\omega}}}\right) \\
=2 R_{m}-2 R_{m} \sqrt{1-\frac{R_{M}^{2}}{\omega}+\frac{R_{M}^{2} R_{m}^{2} \omega^{\prime}}{2 \omega \sqrt{\omega^{2}-R_{M}^{2} \omega}}}
\end{gathered}
$$

So $\left.\frac{\mathrm{d}^{2} g}{\mathrm{~d} R_{m}^{2}}\right|_{R_{m}=0}=0$

$$
\begin{aligned}
\frac{\mathrm{d}^{3} g}{\mathrm{~d} R_{m}^{3}}= & 2-2\left[\sqrt{1-\frac{R_{M}^{2}}{\omega}}+R_{m} \frac{-\frac{R_{M}^{2}}{\omega^{2}} \omega^{\prime}}{2 \sqrt{1-\frac{R_{M}^{2}}{\omega}}}\right] \\
& +\frac{R_{M}^{2}}{2} \frac{\left(2 R_{m} \omega^{\prime}+2 R_{m}^{2} \omega^{\prime \prime}\right) \omega \sqrt{\omega^{2}-R_{M}^{2} \omega}-R_{m}^{2} \omega^{\prime}\left[\sqrt{\omega^{2}-R_{M} \omega}+\frac{\omega\left(2 \omega \omega^{\prime}-R_{M}^{2} \omega^{\prime}\right)}{2 \sqrt{\omega^{2}-R_{M}^{2} \omega}}\right]}{\omega^{2}\left(\omega^{2}-R_{M}^{2} \omega\right)} \\
= & 2-2 \sqrt{1-\frac{R_{M}^{2}}{\omega}}+\frac{R_{m} R_{M}^{2} \omega^{\prime}}{\omega \sqrt{\omega^{2}-R_{M}^{2}}}+\frac{R_{M}^{2}\left(4 R_{m} \omega^{\prime} \omega^{3}-4 R_{m} R_{M}^{2} \omega^{\prime} \omega^{2}+2 \omega^{3} \omega^{\prime \prime} R_{m}^{2}-2 R_{m}^{2} R_{M}^{2} \omega^{\prime \prime} \omega^{2}-2 R_{m}^{2} \omega^{\prime} \omega\right)}{4 \omega^{2}\left(\omega^{2}-R_{M}^{2} \omega\right)^{3 / 2}} \\
& +\frac{R_{M}^{2}\left(2 R_{m}^{2} R_{M}^{2} \omega^{\prime} \omega-2 \omega^{\prime 2} \omega^{2} R_{m}^{2}+R_{m}^{2} R_{M}^{2} \omega^{\prime 2} \omega\right)}{4 \omega^{2}\left(\omega^{2}-R_{M}^{2} \omega\right)^{3 / 2}}
\end{aligned}
$$

where:

$$
\begin{gathered}
\omega^{\prime}=\frac{\mathrm{d} \omega}{\mathrm{d} R_{m}} \text { and } \omega^{\prime \prime}=\frac{\mathrm{d}^{2} \omega}{\mathrm{d} R_{m}^{2}} \\
\text { So }\left.\frac{\mathrm{d}^{3} g}{\mathrm{~d} R_{m}^{3}}\right|_{R_{m}=0}=2-2 \sqrt{1-\frac{R_{M}^{2}}{r^{2}}}
\end{gathered}
$$

Take approximate value (the first item isn't zero)

$$
\begin{aligned}
g\left(R_{m}\right) & \approx g(0)+\frac{\frac{\mathrm{d} g(0)}{\mathrm{d} R_{m}}}{1 !} R_{m}+\frac{\frac{\mathrm{d}^{2} g(0)}{\mathrm{d} R_{m}^{2}}}{2 !} R_{m}^{2}+\frac{\frac{\mathrm{d}^{3} g(0)}{\mathrm{d} R_{m}^{3}}}{3 !} R_{m}^{3} \\
& =0+0+0+\frac{2}{3 !}\left(1-\sqrt{1-\frac{R_{M}^{2}}{r^{2}}}\right) R_{m}^{3}=\frac{1}{3}\left(1-\sqrt{1-\frac{R_{M}^{2}}{r^{2}}}\right) R_{m}^{3}
\end{aligned}
$$


Put (3) into (1)

$$
\begin{aligned}
F_{M} & =\pi a\left(1-\alpha_{1 m}\right) \alpha_{2 M} \rho_{m} f \int_{0}^{\pi} \sin \theta g\left(R_{m}\right) \mathrm{d} \theta \\
& \approx \pi f a\left(1-\alpha_{1 m}\right) \alpha_{2 M} \rho_{m} \int_{0}^{\pi} \frac{\sin \theta}{3}\left(1-\sqrt{1-\frac{R_{M}^{2}}{r^{2}}}\right) R_{m}^{3} \mathrm{~d} \theta \\
& =\frac{2 \pi}{3} f a\left(1-\alpha_{1 m}\right) \alpha_{2 M} \rho_{m}\left(1-\sqrt{1-\frac{R_{M}^{2}}{r^{2}}}\right) R_{m}^{3}
\end{aligned}
$$

Use Taylor expansion

$$
(1+x)^{a}=1+a x+\frac{a(a-1)}{2 !} x^{2}+\cdots \quad|x|<1
$$

Expand $\sqrt{1-\frac{R_{M}^{2}}{r^{2}}}$ and take the first two items, it is obtained:

$$
\sqrt{1-\frac{R_{M}^{2}}{r^{2}}} \approx 1-\frac{1}{2} \frac{R_{M}^{2}}{r^{2}} \quad\left(\because \frac{R_{M}}{r} \ll 1\right)
$$

Put (6) into (4)

$$
\begin{aligned}
F_{M} & \approx \frac{\pi}{3} f a\left(1-\alpha_{1 m}\right) \alpha_{2 M} \rho_{m} \frac{R_{M}^{2} R_{m}^{3}}{r^{2}} \\
& =\frac{\pi}{3} f a\left(1-\alpha_{1 m}\right) \alpha_{2 M} \frac{m M}{\left(\frac{4}{3} \pi\right)^{2} R_{M} \rho_{M} r^{2}} \\
& =\frac{3 f a}{16 \pi}\left(\frac{\left(1-\alpha_{1 m}\right) \alpha_{2 M}}{R_{M} \rho_{M}}\right) \frac{m M}{r^{2}}
\end{aligned}
$$

Method 2:

Set:

$$
\left\{\begin{array}{l}
g\left(R_{m}, R_{M}\right)=\int_{0}^{R_{m}}\left(1-\sqrt{1-\frac{R_{M}^{2}}{\omega}}\right) r_{2}^{2} \mathrm{~d} r_{2} \\
\omega\left(r_{2}\right)=r^{2}+r_{2}^{2}-2 r r_{2} \cos \theta
\end{array}\right.
$$

Under the condition of $r \gg R_{M}+R_{m}$, based on the formula

$$
\begin{aligned}
g(x, y)= & g(0,0)+\frac{1}{1 !}\left(x \frac{\partial}{\partial x}+y \frac{\partial}{\partial y}\right) g(0,0)+\frac{1}{2 !}\left(x \frac{\partial}{\partial x}+y \frac{\partial}{\partial y}\right)^{2} g(0,0) \\
& +\frac{1}{3 !}\left(x \frac{\partial}{\partial x}+y \frac{\partial}{\partial y}\right)^{3} g(0,0)+\cdots
\end{aligned}
$$

Expand the formula and take the first item which is not zero, and is obtained:

$$
F_{M} \approx \frac{3 a f}{16 \pi}\left(\frac{\left(1-\alpha_{1 m}\right) \alpha_{2 M}}{R_{M} \rho_{M}}\right) \cdot \frac{m M}{r^{2}}
$$

Compare (7), (8) with $F=G \frac{m M}{r^{2}}$, it is obtained

$$
G_{M}=\frac{3 a f\left(1-\alpha_{1 m}\right) \alpha_{2 M}}{16 \pi R_{M} \rho_{M}}
$$


Based on a similar deduction process, the following formulas may be got.

$$
F_{m}=\pi a\left(1-\alpha_{1 M}\right) \alpha_{2 m} \rho_{M} f \int_{0}^{\pi} \sin \theta \mathrm{d} \theta \int_{0}^{R_{M}}\left(1-\sqrt{1-\frac{R_{m}^{2}}{r^{2}+r_{2}^{2}-2 r r_{2} \cos \theta}}\right) r_{2}^{2} \mathrm{~d} r_{2}
$$

Which can be simplified as:

$$
\begin{gathered}
F_{m} \approx \frac{3 a f}{16 \pi} \cdot\left(\frac{\alpha_{2 m}\left(1-\alpha_{1 M}\right)}{R_{m} \rho_{m}}\right) \frac{M m}{r^{2}} \\
G_{m}=\frac{3 a f}{16 \pi} \cdot\left(\frac{\alpha_{2 m}\left(1-\alpha_{1 M}\right)}{R_{m} \rho_{m}}\right)
\end{gathered}
$$

where, $\alpha_{2 m}$ is the total absorption coefficient of $m$, and refer to the definition of $\alpha_{2 M}$ for its definition. $\alpha_{1 M}$ is the self-absorption coefficient of $M$, and refers to the definition of $\alpha_{1 m}$ for its definition.

\section{Conclusions}

1) Compatibility between macrophysics and microphysics

This paper applies basic concepts compatible with microphysics, proposes several hypotheses, theoretically derives static-state gravitation formula and establishes a new macrophysics theory. Therefore, a bridge connecting macrophysics and microphysics is built, for reconstructing current macrophysics and microphysics theory, and further exploring natural laws and a new thought.

2) Inverse square law

From the above derivation, it is acquired:

$$
F_{M}=\pi a\left(1-\alpha_{1 m}\right) \alpha_{2 M} \rho_{m} f \int_{0}^{\pi} \sin \theta \mathrm{d} \theta \int_{0}^{R_{m}}\left(1-\sqrt{1-\frac{R_{M}^{2}}{r^{2}+r_{2}^{2}-2 r r_{2} \cos \theta}}\right) r_{2}^{2} \mathrm{~d} r_{2}
$$

Under the condition of $r \gg R_{M}+R_{m}$, the following formula can be obtained through Taylor expansion and taking the first item which is not zero

$$
\begin{gathered}
F_{M} \approx \frac{3 f a}{16 \pi}\left(\frac{\left(1-\alpha_{1 m}\right) \alpha_{2 M}}{R_{M} \rho_{M}}\right) \frac{m M}{r^{2}} \\
\text { So: } F_{M} \propto \frac{1}{r^{2}}
\end{gathered}
$$

Only with $G_{M}=\frac{3 a f\left(1-\alpha_{1 m}\right) \alpha_{2 M}}{16 \pi R_{M} \rho_{M}}$, a formula that is the same as Newton's universal gravitation formula be obtained. Therefore, any experimental results about gravitation which can be reasonably explained by Newton's gravitation theory can also be reasonably explained here. Of course, the difficulties in Newton's gravitation theory can be overcome here; the same as with cosmology.

3) Problems relating to cosmology

As stated above, both Newton's classical gravitation theory and Einstein's general theory of relativity have difficulties and limited in a finite universe. Einstein said the static state of "cosmic space" leads to its close. Actually, it isn't right, because it is based on the old gravitation theory.

This paper starts from the perspective of interaction of field quantum and derives $G_{M}$ and $G_{m}$. therefore, the problem discussed in Section 2 of the part 1 is revised as follows:

The number of lines of force passing through spherical surface $S$ (set as $M$ ) and entering into a sphere directly is not only proportional to $\rho V$ (set as $\rho_{M} \cdot V_{M}$ ), but also proportional to $G_{M} \cdot \rho_{M} \cdot V_{M}$. And as for unit sphere area, the number of lines of force (the field intensity of acting force on a sphere) entering into a sphere is directly proportional to $G_{M} \cdot \rho_{M} \cdot V_{M} / S$ or $G_{M} \cdot \rho_{M} \cdot R_{M}$. 


$$
\text { Here : } G_{M} \rho_{M} R_{M}=\frac{3 a f\left(1-\alpha_{1 m}\right) \alpha_{2 M}}{16 \pi R_{M} \rho_{M}} \rho_{M} R_{M} \propto \frac{1}{\rho_{M} R_{M}} \rho_{M} R_{M}=\text { constant }
$$

Therefore, when $R_{M} \rightarrow \infty$, the field intensity of the acting force on a sphere is not infinitely great, the difficulty caused naturally disappears. This avoids the difficulties that Newton's, Einstein's and other researchers' theories had in this aspect (the problem of the general theory of relativity can also be solved, for example, in $R^{2}=\frac{2}{k \rho}$, by replacing $k$ with $G_{M}^{2}$ ).

Therefore, according to the derivation of this paper, it is concluded that the universe needs not to have started from a big bang, i.e., the universe actually changes in a steady (static)-state.

4) Acting force and counter-acting force

From Newton's age till now, it is admitted that:

a) Under universal gravitation, an acting force is equal to a counter-acting force, i.e. $F_{M}=F_{m}$;

b) Under Coulomb law, the acting force is equal to counter-acting force, i.e. $F_{q_{1}}=F_{q_{2}}$.

However, Newton's Third Law of Motion has its own limits and thus cannot be arbitrarily promoted. It can be seen from the above derivations:

$$
\begin{aligned}
G_{M} & =\frac{3 a f\left(1-\alpha_{1 m}\right) \alpha_{2 M}}{16 \pi R_{M} \rho_{M}} \\
G_{m} & =\frac{3 a f \alpha_{2 m}\left(1-\alpha_{1 M}\right)}{16 \pi R_{m} \rho_{m}}
\end{aligned}
$$

There is no reason to require that $G_{M}$ must be equal to $G_{m}$. In contrast, only when $R_{M}=R_{m}$ and $\rho_{M}=\rho_{m}$, and the objects have the same features, can $G_{M}$ equal to $G_{m}$, which means that in general $F_{M}$ may not equal to $F_{m}$. Only when the gravitons given off by $M$ are all absorbed by $m$, and gravitons given off by $m$ are all absorbed by $M$; or, when the number of gravitons absorbed by $M$ and $m$ are the same, can the acting force be equal to the counter-acting force. In daily life, under the direct interaction between two objects, the acting force is basically equal to the counter-acting force; however, under indirect contact, an acting force may not be equal to a counter-acting force under the interaction of field quantum.

5) Universal gravitation approximation near $M$ surface and extra-small $m$

When near to the $M$ surface, and $m$ is extra small compared with $M$, the approximation conditions between $M$ and $m$ are as follows:

$$
\left\{\begin{array}{l}
R_{m} \ll R_{M} \\
r \approx R_{M}+R_{m} \approx R_{M}
\end{array}\right.
$$

It can be acquired from the above derivations:

$$
F_{M}=\pi a\left(1-\alpha_{1 m}\right) \alpha_{2 M} \rho_{m} f \int_{0}^{\pi} \sin \theta \mathrm{d} \theta \int_{0}^{R_{m}}\left(1-\sqrt{1-\frac{R_{M}^{2}}{r^{2}+r_{2}^{2}-2 r r_{2} \cos \theta}}\right) r_{2}^{2} \mathrm{~d} r_{2}
$$

Under the above approximation conditions, $r_{2}<R_{m} \ll r$, and put it into the above formula:

$$
\begin{aligned}
F_{M} & \approx \pi a\left(1-\alpha_{1 m}\right) \alpha_{2 M} \rho_{m} f \int_{0}^{\pi} \sin \theta \mathrm{d} \theta \int_{0}^{R_{m}}\left(1-\sqrt{1-\frac{R_{M}^{2}}{R_{M}^{2}}}\right) r_{2}^{2} \mathrm{~d} r_{2} \\
& =\pi a\left(1-\alpha_{1 m}\right) \alpha_{2 M} \rho_{m} f \cdot 2 \cdot \frac{1}{3} R_{m}^{3} \\
& =\frac{1}{2} a\left(1-\alpha_{1 m}\right) \alpha_{2 M} f m
\end{aligned}
$$

If $M$ is the earth, and $m$ is a object on earth, to measure the weight of $m$ is to measure the acting force $F_{M}$ of $m$ on measuring tools. In addition, compared with the gravity formula, it is acquired that gravitational acceleration $g$ on earth is: 


$$
g=\frac{1}{2} a\left(1-\alpha_{1 m}\right) \alpha_{2 M} f
$$

Due to $m$ is small, $\alpha_{1 m} \ll 1$, so

$$
g \approx \frac{1}{2} a \alpha_{2 M} f
$$

From the formula of gravitational acceleration $g$, it can be seen that $g$ is related with the absorptive characteristics of earth, and not related to the size and characteristics of $\mathrm{m}$. It is consistent with current experiments and people's common sense, which indicates that the above inference is reasonable, and the formula above also provides a method for measuring the absorption coefficient of gravitons outside of the earth.

If $M$ is big enough to absorb all gravitons given off, so $\alpha_{2 M} \approx 1$ and:

$$
g \approx \frac{1}{2} a f
$$

Physical meaning: when $M$ is big enough, the gravitational acceleration measured on its surface is half of the probability of a unit mass object giving off gravitons multiplied by the equivalent force of a graviton.

6) Difficulties in universal gravitation model

In this model, all basic acting forces should be repulsive forces only from the perspective of momentum and energy conservation, but actually universal gravitation is gravitation. From the perspective of energy conservation, the action process of gravitation should be an exothermic process. For translating the action process of repulsive forces hidden on the surface of a model into the action process of gravitation, there are two probabilities:

a) Translation of universal gravitation, electromagnetic force and strong force

Field quantum is required to release other heavy acting forces in the graviton absorption process (electromagnetic force and strong force). Universal gravitation, electromagnetic force and strong force can translate with each other. This idea may cause further theoretical difficulties.

b) Annihilation effect among gravitons exists in the action process of universal gravitation (more likely)

If an annihilation effect exists among gravitons which contribute to the gravitation effect, and the direction of giving off particles has great changes with annihilation, gravitons may not be absorbed by the object studied. In a gravitation process, gravitons absorbed by objects decrease due to annihilation effects, as a result of which the direction of the resultant force may point in the opposite direction of the vector sum of gravitons less absorbed, which is generally called gravitation direction. If it is true, it will not violate momentum and energy conservation, and cause gravitation effect among the objects studied. In the model, the gravitons absorbed by objects which are mentioned above shall be understood as the effective gravitons influencing gravitation effect, i.e. annihilating gravitons causing an annihilation effect.

Predictions are boldly made on the features of annihilating gravitons and their annihilation effect:

a) Due to weak universal gravitation, the energy generating radiation must be low in the annihilation effect among gravitons.

b) The give-off direction of annihilating particles is different from the collision direction of gravitons, or it is nothing to do with the gravitation effect.

c) Due to the earth is approximately a sphere, the density generated in annihilating particles is around the earth and tends to be approximate, i.e. showing isotropic.

d) When far away from the earth (or other big planets), the density of annihilating particles will decrease accordingly.

The figure (Figure 6) can be proved in the power line figure of actions among charges of same and different polarity. Repulsive force action is elastic scattering while gravitation action causes annihilation effect.

7) Predictions on other secondary effects.

a) The heat in the earth center is provided by universal gravitation, and the heat released is related to the position of the earth in a galaxy (density of planets in surrounding environment), and the change law of earthquake frequency is closely related to the heat release effect of universal gravitation.

b) Temperature change within the earth over long time periods is consistent with the change law of earthquake frequency.

Previous literatures [16] suggested that: The condition that the solar system crosses through the spiral arms of 


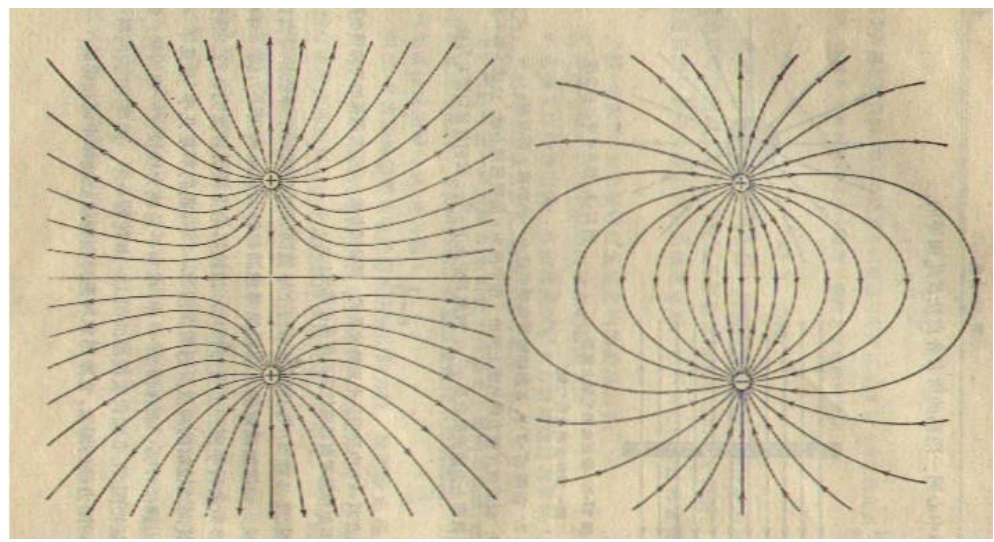

(a)

(b)

Figure 6. Power line of actions among chages of same and different polarity. (a) Like charges repel; (b) opposite charges attract.

a galaxy is involved with that of the paleomagnetic field reversing. Literatures [17]-[19] also found that: paleomagnetic field is closely related to climate factors. This further indicates that universal gravitation is closely related to electromagnetic force and climate change factors.

8) New explanations about $3 \mathrm{k}$ background radiation experimental phenomena which support big bang theory Under the new model, new explanations about $3 \mathrm{k}$ background radiation experimental phenomena which support big bang theory are made:

From the discussion in 5, it is known that under the action of gravitons, if an annihilation effect can be caused, $3 \mathrm{k}$ background radiation may be the secondary product generated in the translation process, and its intensity changes with the distance away from the star. Farther the distance is, lower the intensity of $3 \mathrm{k}$ background radiation. Of course, the distance cannot be too far, or isotropic features of $3 \mathrm{k}$ background radiation will be influenced by near stars. If the big bang theory is right, the intensity of $3 \mathrm{k}$ background radiation will be the same in any position within the solar system.

\section{Acknowledgements}

Here I want to express my sincere gratitude to Dr. Wu Yuejiang of Beijing Normal University for his revision on this paper. I also thank prof. Peter Pearce of CERN, and he took many times to make a few corrections on this paper.

\section{References}

[1] Zhou, P.Y. (1981) Scientia Sinica, No. 4, P334-P345.

[2] See, W.D. (1911) Monthly Notices of the Royal Astronomical Society, 7l, 388.

[3] Lv, J.H. (1984) Journal of Chinese Science and Technology University, No. 1, P22-P26.

[4] Lv, J.H. (1986) Studies in Dialectics of Nature, 2, P27-P34.

[5] French, A.P. (1987) World Science, 3, P25-P28.

[6] Newman, R.D. (1982) Tests of the Gravitational Inverse Square Law on Laboratory Distance Scale. The 3rd Marcel Grossman Meeting on Recent Development in General Relativity, Shanghai, 30 August-3 September 1982, 12 p.

[7] Rosser, W.G.V. (1971) An Introduction to the Theory of Relativity (in Chinese). Butterworths, London. Translated by Yue, Z.Y. and Guan, D.X., Science Press, Beijing.

[8] Einstein, A. (1955) RELATIVITY The Special and The General Theory (in Chinese). Methuen \& Co. Ltd., London. Translated by Yang Runyin, Shanghai Science and Technology Press, Beijing.

[9] Pauli, W. (1958) Theory of Relativity (in Chinese). Pergamon Press, Oxford. Translated by Ling, D.H. and Zhou, W.S., Shanghai Science and Technology Press, Beijign.

[10] Weinberg. S. (1980) Gravitation and Cosmology, Principles and Applications of the General Theory of Relativity (in Chinese). Science Press, Beijing. 
[11] Filkin, D. (2000) Stephen Hawking’s Universe. Translated by Zhao, F.H., Hainan Press, Beijing.

[12] Dark Energy, Dark Matter. (2014) http://science.nasa.gov/astrophysics/focus-areas/what-is-dark-energy/

[13] “Dark Energy” and “Dark Matter”. (2014) http://baike.baidu.com/view/50517.htm?fr=wordsearch,http://baike.baidu.com/subview/763/5784093.htm?fromId=763 \&from $=$ rdtself\&fr=wordsearch

[14] John, H. (1997) The End of Science. Yuanfang Press, Beijing.

[15] He, X.T. and Qiao, G. (1993) Studies in Dialectics of Nature, 9, P12-P17.

[16] Li, G.D. (1999) Modern Magnetics. Press of University of Science and Technology of China, Hefei.

[17] Kok, Y.S. (1999) Earth and Planetary Science Letters, 166, 105-119.

[18] Bucha, V. and Bucha Jr., V. (1998) Journal of Atmospheric and Solar-Terrestrial Physics, 60, 145-169. http://dx.doi.org/10.1016/S1364-6826(97)00119-3

[19] Guyodo, Y., Richter, C. and Valet, J.P. (1999) Journal of Geophysical Research, 104, 22953-22964. http://dx.doi.org/10.1029/1999JB900163 
Scientific Research Publishing (SCIRP) is one of the largest Open Access journal publishers. It is currently publishing more than 200 open access, online, peer-reviewed journals covering a wide range of academic disciplines. SCIRP serves the worldwide academic communities and contributes to the progress and application of science with its publication.

Other selected journals from SCIRP are listed as below. Submit your manuscript to us via either submit@scirp.org or Online Submission Portal.
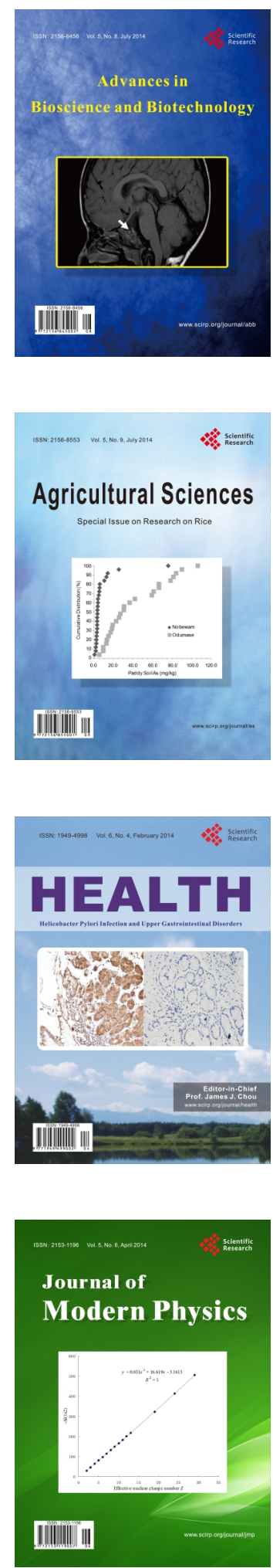
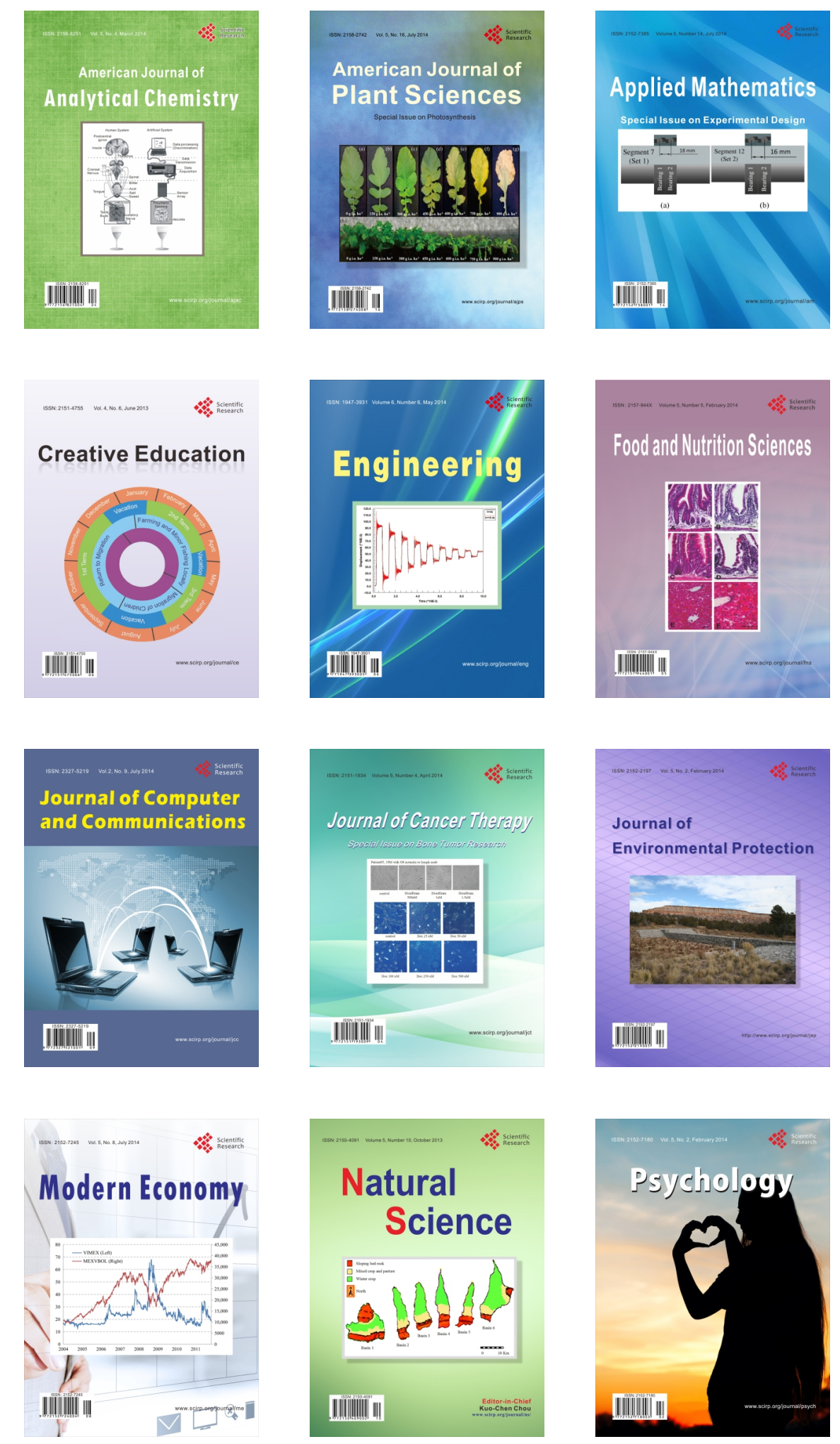